\title{
Congenital hydrocephalus secondary to Walker-Warburg syndrome identified on the Manitoba Neonatal Screening Programme for Duchenne Muscular Dystrophy
}

\author{
C R Greenberg, H K Jacobs, T E Nylen, M Gibb, B N Chodirker, M Moffatt, \\ A Lacson, W Halliday, F Bernier, A El-Husseini, A Cameron, $\mathrm{K}$ Wrogemann
}

\section{Department of \\ Pediatrics and Child Health, University of Manitoba, Winnipeg, Manitoba, Canada. C R Greenberg $M$ Gibb B N Chodirker M Moffatt F Bernier}

Department of Human Genetics, University of Manitoba, 250-770 Bannatyne Avenue, Winnipeg, Manitoba R3E 0W3, Canada.

C R Greenberg

M Gibb

B N Chodirker

F Bernier

Department of Pathology, University of Manitoba Winnipeg, Manitoba, Canada.

A Lacson

W Halliday

Department of Biochemistry and Molecular Biology, University of Manitoba, Winnipeg Manitoba, Canada.

H K Jacobs

T E Nylen

A El-Husseini

A Cameron

$\mathrm{K}$ Wrogemann

Correspondence to Dr Greenberg.

Received 20 June 1991.

Revised version accepted

31 January 1992.

\begin{abstract}
This report describes our first experience with a clinically important true false positive neonatal screening test for Duchenne muscular dystrophy (DMD). Neonatal screening for DMD began as a pilot programme in Manitoba on 1 January 1986 by analysis of creatine kinase (CK) activity in dried filter paper blood spots. To date, all except two males with positive initial and follow up neonatal CK screening tests were subsequently diagnosed as having DMD. Of these two, one was a newborn male with congenital hydrocephalus whose positive DMD screening test led to the identification of an associated congenital myopathy and confirmation of the diagnosis of WalkerWarburg syndrome.
\end{abstract}

Between 1 January 1986 and 31 August 1990, 43513 newborn males have been screened for Duchenne muscular dystrophy by analysis of creatine kinase (CK) activity in dried filter paper blood spots as part of the Manitoba Pilot Neonatal Screening Programme for Duchenne Muscular Dystrophy (DMD) and Becker Muscular Dystrophy (BMD). Eight asymptomatic infant boys have been identified to date with persistently raised $C K$ values and were subsequently diagnosed as having presymptomatic DMD. ${ }^{1}$ Follow up specimens have been received to date on an additional 35 males who had initial positive neonatal CK screening tests. In all but two the CK levels had become normal at the time of repeat sampling. Of these two, one male was shown to have persistent but benign raised CK-BB isoenzyme. ${ }^{2}$ We now report a newborn male with congenital hydrocephalus and cataracts who had persistently high CK levels. The positive neonatal screening test for DMD in this child led to the identification of an associated congenital myopathy and subsequent confirmation of the diagnosis of Walker-Warburg syndrome.

\section{Case report}

A male infant was born to a 21 year old, healthy, G3P2 mother of Inuit and Caucasian ancestry by caesarean section at 41 weeks' gestation because of prenatal identification of congenital hydrocephalus. The identity of the biological father was uncertain. The pregnancy was complicated by a chlamydial infection at 7 months' gestation and the occasional use of marijuana and hashish. Her first pregnancy had resulted in the birth of a healthy daughter and the second pregnancy ended in an early spontaneous miscarriage. One maternal aunt of the proband was known to have Prader-Willi syndrome with a de novo cytogenetic deletion in 15q11-q13. The proband's maternal first cousin died in infancy from complications of well documented neonatal adrenoleucodystrophy.

On examination after birth, gross macrocrania secondary to congenital hydrocephalus was evident with a head circumference of $48 \mathrm{~cm}$, birth weight $4425 \mathrm{~g}$, and birth length $55 \mathrm{~cm}$. No congenital ocular, facial, musculoskeletal, genitourinary, or internal malformations were immediately evident after birth, but cataracts were noted on the second day of life. The fundi could not be visualised. Neurologically the baby was initially very hypotonic with very poor suck and gag reflexes, but he did not require ventilatory assistance. A right ventriculoperitoneal shunt was inserted on the second day of life. He subsequently tolerated tube feedings, responded to noises, cried loudly, and moved spontaneously. His clinical course was marked by repeated cerebrospinal fluid infections necessitating two shunt revisions. He died at 8 months of age. Permission for necropsy was not obtained. No meaningful social or motor development had been evident.

Chromosome analysis showed a normal male karyotype, 46,XY. TORCH screen was negative. The Pilot Neonatal Screening Programme for Duchenne Muscular Dystrophy reported a grossly raised creatine kinase (CK) level using a modified fluorescent assay performed on filter paper blood spots collected in the routine Manitoba Perinatal Screening Programme as previously described. ${ }^{2} \mathrm{~A}$ repeat filter paper assay showed a persistently raised CK level with CK-MM being the predominant isozyme. Two venous $C K$ levels performed at 3 months of age were 3500 and $5368 \mathrm{U} / 1$ respectively (normal $<180 \mathrm{U} / \mathrm{l}$ ). Electromyographic (EMG) examination was consistent with a myopathic process. Ultrasound and CT scan of the head obtained post shunt showed marked dilatation of both lateral, third, and fourth ventricles. Lack of surface gyral markings favoured the diagnosis of lissencephaly (fig 1) and posterior fossa images suggested 


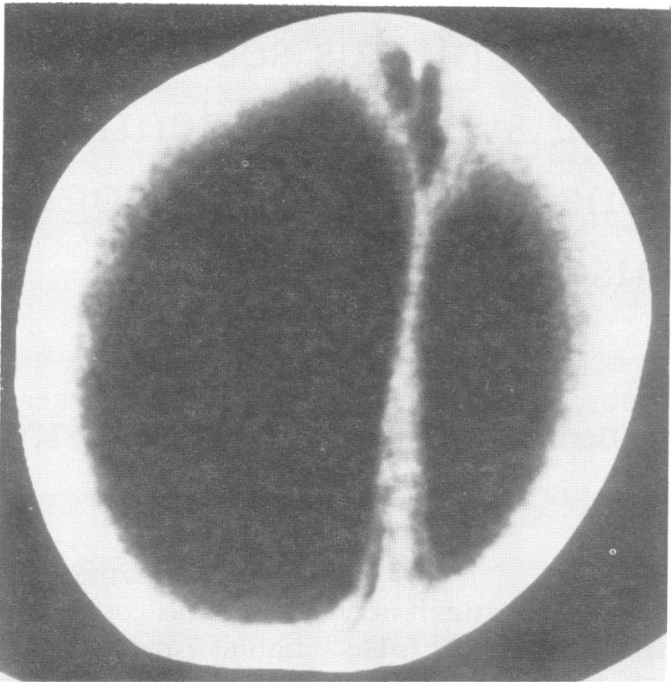

Figure 1 CT scan of the head post shunt showing marked dilatation of the lateral ventricles and lack of surface gyral markings suggesting type II lissencephaly.

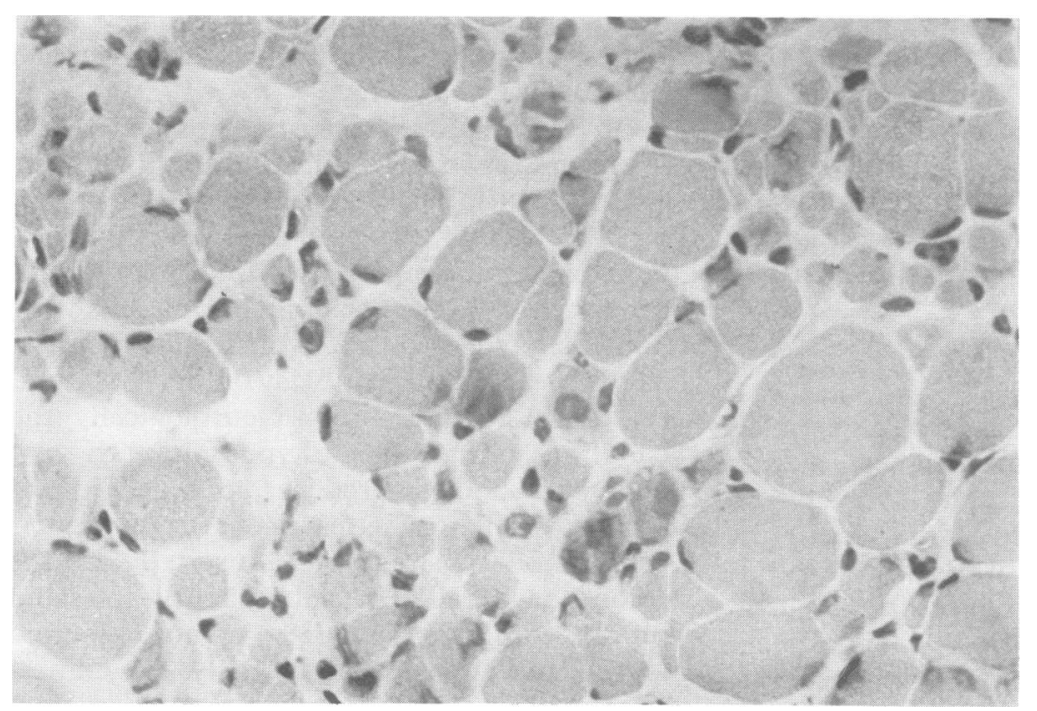

Figure 2 The muscle biopsy features marked random fibre size variation, regenerating fibres, and so-called 'myopathic groups' of small fibres (HE्E).

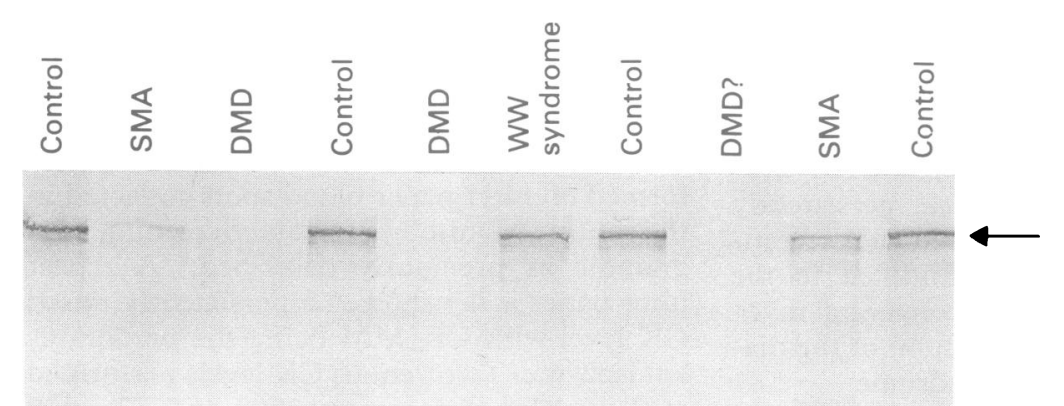

Figure 3 Western blot analysis for dystrophin. Dystrophin is readily detectable in our patient (lane 6), controls (lanes 1, 4, 7, 10), and patients with spinal muscular atrophy (lanes 2 and 9). Dystrophin is not detected in patients with known (lanes 3 and 5) and suspected DMD (lane 8). cerebellar hypoplasia. Ultrasound of the orbits showed a nodular density in the left orbit anterior to the optic nerve consistent with the presence of a left retinal detachment. A muscle biopsy was obtained from his left quadriceps muscle at $3 \frac{1}{2}$ months of age and showed evidence of an active dystrophic process with excessive variation in fibre size, fibre necrosis, and regeneration as well as focal fat replacement (fig 2). Western blot analysis of muscle dystrophin using sheep antidystrophin antisera (courtesy of Dr L Kunkel and co-workers) and mouse monoclonal antidystrophin antibodies to the rod portion of dystrophin (NCLDYS1, Novacastra Laboratories, Newcastle) (fig 3) showed abundant dystrophin expression. ${ }^{3}$ Immunohistochemical analysis of dystrophin expression confirmed abundant dystrophin staining (data not shown). No detectable alterations were evident in the dystrophin gene using polymerase chain reaction multiplex amplification of eight exons ${ }^{4}$ and Southern blot analysis using six separate ${ }^{32} \mathrm{P}$ labelled cDNAs from the American Type Tissue Collection (ATTC, Rockville, Md) that identify over 60 exons spanning the 2 megabase dystrophin gene. ${ }^{5}$

\section{Discussion}

We propose that our patient had the WalkerWarburg syndrome (WWS) (MIM reference 236670), an autosomal recessive syndrome characterised by severe brain and eye malformations. ${ }^{67}$ Type II lissencephaly, cerebellar malformation, and retinal malformation have been present in all reported cases but recently the Walker-Warburg phenotype has been expanded to include congenital muscular dystrophy as a major diagnostic criterion. ${ }^{7}$ Documented muscle changes vary widely but all reports include variability of fibre size, central nuclei, fibre splitting, necrosis, and fat infiltration consistent with congenital muscular dystrophy. ${ }^{7}$ Although clinical similarities exist with Fukuyama congenital muscular dystrophy, such severe eye abnormalities are uncommon in the latter disorder and it is rarely diagnosed outside Japan. ${ }^{89}$ Necessary diagnostic criteria have included type II lissencephaly, cerebellar malformation, and retinal malformation. Raised serum CK has been noted in virtually all patients reported, but the rise in CK varies greatly both between patients ( 3 to 60 times the predicted normal values) and over time in any individual patient. ${ }^{7}$ The identification on a neonatal screening programme of raised $\mathrm{CK}$ in this patient led to the assay of the venous $C K$, the EMG examination, and ultimately to his muscle biopsy which showed active dystrophic pathological changes. The positive identification of congenital muscular dystrophy allowed us to confirm the clinical diagnosis of Walker-Warburg syndrome in this child whose other findings including congenital hydrocephalus, and strong suspicion of type II lissencephaly, retinal malformation, and congenital cataracts by themselves were 
highly suggestive of the Walker-Warburg syndrome. The underlying cause and pathogenesis of the dystrophic muscle seen in WalkerWarburg syndrome is unknown, but we clearly found in this report that it is unrelated to dystrophin deficiency. Whether other dystrophin-like gene products are implicated remains to be determined.

Our pilot neonatal screening programme for $\mathrm{DMD} / \mathrm{BMD}$ is designed to test the hypothesis that very early identification of DMD boys and subsequent identification of carrier women in their families will lead to a decreased population incidence of DMD and BMD. Approximately 1 in 1000 children have initial positive CK screening tests that require repeat sampling. One child to date has been identified as having persistently raised CK-BB, a benign red cell abnormality. Persistently raised CKMM on repeat filter paper assay had to date only been seen in our eight true DMD/BMD positives. ${ }^{1}$ The ascertainment of this child with WWS represents a true false positive. It is thus now important to note that other forms of congenital myopathic disorders, including WWS, may be identified on neonatal screening programmes for DMD and BMD. No other male with WWS has been identified clinically between the time of beginning neonatal DMD screening and the ascertainment of this child. In that time period around 50000 males have been screened and an additional two females with WWS have been diagnosed. This ascertainment of three cases of WWS in 100000 livebirths suggests a minimal population frequency of $\sim 1 / 33333$ for this disorder. Since the institution of the Manitoba pilot screening programme, four other males known to us presented in the neonatal period and were subsequently diagnosed as having congenital myopathic disorders (one idiopathic congenital muscular dystrophy, one $\mathrm{X}$ linked myotubular myopathy, one phosphofructokinase deficiency, one minicore myopathy). These boys did not have positive neonatal $\mathrm{CK}$ screening tests presumably reflecting their only minimally raised venous $\mathrm{CK}$. Nonetheless, we conclude that in addition to true cases of DMD and BMD being ascertained on our neonatal screening programme, other forms of congenital muscular dystrophy may be identified and should be considered when persistently raised $C K$ is detected.

We gratefully acknowledge the assistance of the medical and nursing staff of the Children's Hospital, the support of the nursing and interpreter staff of the J A Hildes Northern Medical Unit, the receipt of the PCR multiplex kits from Drs Tom Caskey and Richard Gibbs (Houston), and the expert secretarial assistance of Josie Diato. This work was supported by the Muscular Dystrophy Association of Canada and the Paul H T Thorlakson Research Foundation.

1 Greenberg CR, Jacobs HK, Halliday W, Wrogemann K. Three years' experience with neonatal screening for Three years experience with neonatal screening for Duchenne/Becker muscular dystrophy: gene analysis, gene 1991;39:68-75.

2 Jacobs HK, Wrogemann K, Greenberg CR, Seshia SS, Cameron AI. Neonatal screening for Duchenne muscular dystrophy - the Canadian experience. In: Schmidt BJ Diament AJ, Loghin-Grosso NS, eds. Current trends in infant screening. Amsterdam: Elsevier, 1989:361-6.

3 Hoffman EP, Fischbeck KH, Brown RH, et al. Characterization of dystrophin in muscle biopsy specimens from patients with Duchenne's or Becker's muscular dystrophy. N Engl f Med 1988;318:1363-8.

4 Chamberlain JS, Gibbs RA, Ravier JE, Nguyen PN, Caskey CT. Deletion screening of the Duchenne muscular dystrophy locus via multiplex DNA amplification. Nucleic trophy locus via multiplex

5 Koenig M, Hoffman EP, Bertelson CJ, Monaco AP, Feener C, Kunkel LM. Complete cloning of the Duchenne muscular dystrophy (DMD) cDNA and preliminary organization of the DMD gene in normal and affected individuals. Cell 1987;50:509-17.

6 Whitley CB, Thompson TR, Mastri AR, Gorlin RJ Warburg syndrome: lethal neurodysplasia with autosomal recessive inheritance. $\mathcal{F}$ Pediatr 1983;102:547-51.

7 Dobyns WB, Pagon RA, Armstrong D, et al. Diagnostic criteria for Walker-Warburg syndrome. Am $f$ Med Genet 1989;32:195-210.

8 Fukuyama Y, Ohsawa M. A genetic study of the Fukuyama type of congenital muscular dystrophy. Brain Dev 1984;6:373-90.

9 McMenamin JB, Becker LE, Murphy EG. Congenital muscular dystrophy: a clinicopathologic report of 24 cases. $\mathcal{J}$ Pediatr 1982;100:692-7. 